\title{
NONCOMMUTATIVE EXTENSION OF AN INTEGRAL REPRESENTATION THEOREM OF ENTROPY
}

\author{
Dedicated to Professor H. Umegaki on his 60th birthday
}

\author{
BY NOBORU WATANABE
}

\section{Introduction}

In 1964, Umegaki proved a theorem of McMillan type concerning the integral representation of entropy in the measure theoretic framework, about which we briefly review in $\S 1$. Noncommutative probability theory is important to analyse some physical systems $[1,2,4,5,6,7,10,11,12,13,16,17]$. In this paper, using various results obtained in operator algebras, we extend this theorem to that for noncommutative systems.

\section{§1. Integral representation of entropy}

Let $X$ be a compact metric space and $\mathfrak{B}(X)$ be the $\sigma$-field of all Borel sets in $X$. We denote a homeomorphism on $X$ by $T$ and the set of all $T$-invariant regular probability measures $p, q, \cdots$ on $X$ by $P_{T}$. Let $\mathcal{P}$ be a finite partition of $X$ and we put $\mathfrak{M}_{n}=\bigvee_{k=1}^{n} T^{-k} \mathscr{P}$ and $\mathfrak{M}_{\infty}=\bigvee_{k=1}^{\infty} \mathfrak{M}_{k}$. Then the entropy of each $p \in P_{T}$ is defined by

$$
S(p)=-\lim \frac{1}{n} \Sigma_{U} p(U) \log p(U) \quad(n \rightarrow \infty),
$$

where $\Sigma_{U}$ means the summation over $U$ of the atomic sets in $\mathscr{Q} \vee \mathfrak{M}_{n-1}$. For any $p \in P_{T}$, we denote the conditional probability functions of $U \in \mathscr{P}$ with respect to $\mathfrak{M}_{n}$ and $\mathfrak{M}_{\infty}$ by $P_{p}\left(U \mid \mathfrak{M}_{n}\right)$ and $P_{p}\left(U \mid \mathfrak{M}_{\infty}\right)$ respectively. Now we define the $\mathfrak{M}_{\infty}$-measurable function $h_{p}(x)$ on $X$ as follows:

$$
h_{p}(x)=-\sum_{U \in \mathcal{P}} P_{p}\left(U \mid \mathfrak{M}_{\infty}\right) \log P_{p}\left(U \mid \mathfrak{M}_{\infty}\right)(x) \quad \text { p-a. e. } \quad x \in X,
$$

for any $p \in P_{T}$. Then, the next important theorem [14] of McMillan type holds.

THEOREM 1. For any finite partition $\mathcal{Q}$, there universally exists a Borel measurable function $h(x)$ on $X$ such that it is bounded, non-negative, T-invariant and satisfies

(1) $h(x)=h_{p}(x)$ p-a.e. $x \in X$ and for every $p \in P_{T}$,

Received June 20, 1985 
(2) $S(p)=\int_{X} h(x) d p(x)$ for every $p \in P_{T}$.

A typical example of $X$ is a compact message space $A^{Z}$, where $A$ is a set of some alphabets [15]. Then $T$ is the shift in $A^{Z}$. This case provides with a concrete description of communication processes.

\section{§2. Noncommutative extension}

Let $B(\mathscr{H})$ be the set of all bounded operators on a separable Hilbert space $\mathscr{H}$ and let $\mathfrak{N}$ be a von Neumann algebra (i. e. $\mathfrak{N}=\left(\mathfrak{N}^{\prime}\right)^{\prime}$ where $\mathfrak{N}^{\prime}=\{A \in B(\mathscr{H})$; $A B-B A=0$ for any $B \in \mathfrak{R}\})$ acting on $\mathscr{H}$. In noncommutative systems, we use a *-automorphism $\alpha$ of $\mathfrak{R}$ instead of $T$. We further denote the set of all finite partitions of $I$ in $\mathfrak{N}$ by $\mathscr{Q}(\mathfrak{N})$ (i. e. $\widetilde{P}=\left\{P_{j} ; j=1,2, \cdots, n<\infty\right\} \in \mathscr{P}(\mathfrak{N})$ satisfies (i) $P_{\jmath} \perp P_{k}(k \neq j)$ and (ii) $\left.\sum_{j=1}^{n} P_{\jmath}=I\right)$.

We denote the set of all normal states on $\mathfrak{N}$ by $\mathfrak{S}(\mathfrak{N})$ and the set of all $\alpha$-invariant states in $\mathfrak{S}(\mathfrak{R})$ by $\mathfrak{S}_{I}(\alpha)$. We assume that there exists a faithful state in $\mathfrak{S}_{I}(\alpha)$. Let $\mathfrak{M}$ be a von Neumann subalgebra of $\mathfrak{R}$ including $\mathfrak{R}^{\alpha}$, where $\mathfrak{N}^{\alpha} \equiv\{A \in \mathfrak{R} ; \alpha(A)=A\}$, and let $\mathfrak{M}_{n}, \mathfrak{M}_{\infty}$ be the von Neumann subalgebras generated by $\bigcup_{k=1}^{n} \alpha^{k}(\mathfrak{M}), \bigcup_{k=1}^{\infty} \alpha^{k}(\mathfrak{M})$ respectively. For each $\varphi \in \mathfrak{S}_{I}(\alpha)$, we further denote the conditional expectations $[10,17]$ of $A \in \mathfrak{N}$ with respect to $\mathfrak{M}$ and $\mathfrak{M}_{n}$ $(\forall n \in N)$ by $E_{\varphi}(A \mid \mathfrak{M})$ and $E_{\varphi}\left(A \mid \mathfrak{M}_{n}\right)$ respectively. For any faithful $\varphi \in \mathcal{S}_{I}(\alpha)$, let $\left\{\sigma_{t}^{\varphi} ; t \in R\right\}$ be the modular automorphism group $[9,17]$ with respect to $\varphi$ at $\beta=1$. We assume that there exists the conditional expectation $E_{\varphi}(\cdot \mid \mathfrak{M})$ for $\varphi \in \widehat{S}_{I}(\alpha)$. We call this assumption " $\langle A\rangle$ " for $\varphi$ in the sequel.

LEMMA 2. For any faithful $\varphi \in \mathbb{S}_{I}(\alpha)$ with $\langle A\rangle$, there exists the conditional expectation $E_{\varphi}\left(\cdot \mid \mathfrak{M}_{n}\right)$ for any $n \in N$.

Proof. For an $\alpha$-invariant state $\varphi$, we have

$$
\sigma_{t}^{\varphi} \circ \alpha=\alpha \circ \sigma_{t}^{\varphi} \quad \text { for any } t \in R .
$$

When $n=1$, we obtain $\sigma_{t}^{\varphi}\left(\mathfrak{M}_{1}\right)=\sigma_{t}^{\varphi} \circ \alpha(\mathfrak{M})=\alpha \circ \sigma_{t}^{\varphi}(\mathfrak{M})=\alpha(\mathfrak{M})=\mathfrak{M}_{1}$. Suppose that $\sigma_{t}^{\varphi}\left(\mathfrak{M}_{n}\right)=\mathfrak{M}_{n}$ holds for $n \in N$. Then

$$
\begin{aligned}
\sigma_{t}^{\varphi}\left(\mathfrak{M}_{n+1}\right) & =\sigma_{t}^{\varphi}\left(\alpha\left(\mathfrak{M}_{n}\right) \vee \mathfrak{M}_{1}\right) \\
& =\sigma_{t}^{\varphi} \circ \alpha\left(\mathfrak{M}_{n}\right) \vee \sigma_{t}^{\varphi}\left(\mathfrak{M}_{1}\right) \\
& =\alpha\left(\mathfrak{M}_{n}\right) \vee \mathfrak{M}_{1} \\
& =\mathfrak{M}_{n+1} \quad \text { for any } \quad t \in R .
\end{aligned}
$$

Therefore there exists the conditional expectation $E_{\varphi}\left(\cdot \mid \mathfrak{M}_{n}\right)$ for any $n \in N$.

Q.E. D. 
We here remind of two topologies in $B(\mathscr{H})[17]:$ (i) $A$ net $\left\{A_{\alpha}\right\} \subset B(\mathscr{H})$ converges to $A \in B(\mathscr{H})$ in the strong operator topology (write $A_{\alpha} \stackrel{s}{\longrightarrow} A$ ) if $\left\|\left(A_{\alpha}-A\right) x\right\| \rightarrow 0$ for any $x \in \mathscr{H}$, (ii) a net $\left\{A_{\alpha}\right\} \subset B(\mathscr{H})$ converges to $A \in B(\mathscr{H})$ in the ultrastrong operator topology (write $A_{\alpha} \stackrel{u s}{\longrightarrow} A$ ) if $\sum_{n}\left\|\left(A_{\alpha}-A\right) x_{n}\right\|^{2} \rightarrow 0$ for any sequence $\left\{x_{n}\right\} \subset \mathcal{H}$ such that $\Sigma_{n}\left\|x_{n}\right\|^{2}<\infty$.

From the definition of $\mathfrak{M}_{n},\left\{\mathfrak{M}_{n}\right\}$ is an increasing sequence of von Neumann subalgebras. According to Lemma 2, we have (c.f. [11, 17]).

$1^{\circ} \quad E_{\varphi}\left(A \mid \mathfrak{M}_{n}\right) \stackrel{u s}{\longrightarrow} E_{\varphi}\left(A \mid \mathfrak{M}_{\infty}\right)$ for any $A \in \mathfrak{R}$ and any faithful $\varphi \in \mathbb{S}_{I}(\alpha)$ with $\langle A\rangle$.

$\mathfrak{M}$ is said to be a sufficient $[1,2,12,13]$ for $\mathcal{S} \subset \mathcal{S}(\mathfrak{N})$ if $E_{\varphi}(\cdot \mid \mathfrak{M})$ exists for each $\varphi \in \mathcal{S}$ and for each $A \in \mathfrak{N}$ there exists an $A_{0} \in \mathfrak{M}$ such that

$$
A_{0}=E_{\varphi}(A \mid \mathfrak{M}) \quad \varphi \text {-a. e., } \varphi \in \mathcal{S},
$$

where $A=B \varphi$-a.e. means $\varphi(|A-B|)=0$. In [3], Nakamura and Umegaki showed that the function $\eta(A)=-A \log A$ for any positive $A \in \mathfrak{N}$ is operator concave. We assume that $\mathfrak{S}_{I}(\alpha)$ includes a faithful state with $\langle A\rangle$. Using this function $\eta$, we define

$$
S_{\varphi}^{\tilde{P}}\left(\mathfrak{M}_{n}\right) \equiv \Sigma_{j} \eta\left(E_{\varphi}\left(P_{j} \mid \mathfrak{M}_{n}\right)\right)
$$

for any finite partition $\tilde{P}=\left\{P_{\jmath}\right\} \in \mathscr{L}(\mathfrak{M})$ and $\varphi \in \mathfrak{S}_{I}(\alpha)$, which is uniquely determined in the sense of $\varphi$-a.e.. Moreover, we define $S_{\varphi}^{\tilde{P}}$ as follows: For any finite partition $\tilde{P}=\left\{P_{j}\right\} \in \mathscr{P}(\mathfrak{M})$ and $\varphi \in \mathfrak{S}_{I}(\alpha)$,

$$
S_{\varphi}^{\tilde{P}}\left(\mathfrak{M}_{n}\right) \equiv \varphi\left(s_{\varphi}^{\tilde{P}}\left(\mathfrak{M}_{n}\right)\right)=\Sigma_{\jmath} \varphi\left(\eta\left(E_{\varphi}\left(P_{j} \mid \mathfrak{M}_{n}\right)\right)\right)
$$

Then the following lemma holds.

LEMMA 3. For any faithful $\varphi \in \mathbb{S}_{I}(\alpha)$ with $\langle A\rangle$, we obtain

(1) $s_{\varphi}^{\tilde{P}}\left(\mathfrak{M}_{n}\right) \stackrel{s}{\longrightarrow} s_{\varphi}^{\tilde{P}}\left(\mathfrak{M}_{\infty}\right)$

(2) $S_{\varphi}^{\tilde{P}}\left(\mathfrak{M}_{n}\right) \longrightarrow S_{\varphi}^{\tilde{P}}\left(\mathfrak{M}_{\infty}\right) \quad(n \rightarrow \infty)$

for any partition $\tilde{P} \in \mathscr{Q}(\mathfrak{M})$.

Proof. It is known that [8] the convergence $A_{n} \stackrel{s}{\longrightarrow} A$ for a bounded sequence $\left\{A_{n}\right\}$ implies $f\left(A_{n}\right) \stackrel{s}{\longrightarrow} f(A)$ for any continuous function $f(t)$ such that $f(0)=0$ and $|f(t)| \leqq \alpha|t|+\beta$ with positive constants $\alpha$, $\beta$. Since $\eta(t)$ satisfies the above conditions, we obtain on the support of $\varphi$

$$
s_{\varphi}^{\tilde{P}_{\varphi}}\left(\mathfrak{M}_{n}\right) \stackrel{s}{\longrightarrow} s_{\varphi}^{\tilde{P}}\left(\mathfrak{M}_{\infty}\right)
$$

for any partition $\tilde{P} \in \mathscr{P}(\mathfrak{M})$ and any $\varphi \in \mathscr{S}_{I}(\boldsymbol{\alpha})$. (2) is immediate for (1).

Q.E.D. 
THEOREM 4. We assume that $\boldsymbol{S}_{I}(\alpha)$ includes a faithful state with $\langle A\rangle$. Then there exists a positive operator $h(\tilde{P}, \alpha)$ satisfying

(1) $h(\tilde{P}, \alpha)=s_{\varphi}^{\tilde{P}}\left(\mathfrak{M}_{\infty}\right) \quad \varphi-a . e$.

(2) $S_{\varphi}^{\tilde{P}}\left(\mathfrak{M}_{\infty}\right)=\varphi(h(\tilde{P}, \alpha))$

for any partition $\tilde{P} \in \mathcal{Q}(\mathfrak{M})$ and any $\varphi \in \Im_{I}(\alpha)$.

Proof. By Theorem 6.49 of [17] (i. e. if $\mathfrak{S}_{I}(\alpha)$ includes a faithful state, then $\mathfrak{N}^{\alpha}$ is sufficient for $\mathfrak{S}_{I}(\alpha)$ ), $\mathfrak{\Re}^{\alpha}$ is sufficient for $\mathfrak{S}_{I}(\alpha)$. Moreover, the above lemma 2 and the fact $4^{\circ}$ of [1] (i. e. if $\mathcal{S}(\subset \mathcal{S}(\mathfrak{R})$ ) contains a faithful state $\varphi$ and $\mathfrak{M}$ is sufficient for $\mathcal{S}$, then any subalgebra $\mathfrak{M}_{0}$ including $\mathfrak{M}$ is sufficient for $\mathcal{S}$ whenever $E_{\varphi}\left(\cdot \mid \mathfrak{M}_{0}\right)$ exists) imply that $\mathfrak{M}_{n}$ is sufficient for $\mathfrak{S}_{I}(\alpha)(n \in N)$. Let $\phi$ be a faithful state in $\mathfrak{S}_{I}(\alpha)$ with $\langle A\rangle$. Since $\mathfrak{M}_{n}$ is sufficient for $\mathfrak{S}_{I}(\alpha)$, $\varphi \circ E_{\psi}\left(\cdot \mid \mathfrak{M}_{n}\right)=\varphi(\cdot)$ holds for any $\varphi \in \mathfrak{S}_{I}(\alpha)$. By the fact $1^{\circ}$, the sequence $\left\{E_{\psi}\left(\cdot \mid \mathfrak{M}_{n}\right)\right\}$ is strongly convergent to $E_{\psi}\left(\cdot \mid \mathfrak{M}_{\infty}\right)$ satisfying $\varphi^{\circ} E_{\psi}\left(\cdot \mid \mathfrak{M}_{\infty}\right)=\varphi(\cdot)$ for any $\varphi \in \mathfrak{S}_{I}(\alpha)$. Therefore $\mathfrak{M}_{\infty}$ is sufficient for $\mathfrak{S}_{I}(\alpha)$, which implies that there exists the conditional expectation $\xi$ from $\mathfrak{N}$ to $\mathfrak{M}_{\infty}$ such that $\varphi \circ \xi=\varphi$ for any $\varphi \in \mathbb{S}_{I}(\alpha)$. From Lemma 3 , the sequence $\left\{\eta\left(E_{\psi}\left(A \mid \mathfrak{M}_{n}\right)\right)\right\}$ is strongly convergent to $\eta\left(E_{\psi}\left(A \mid \mathfrak{M}_{\infty}\right)\right)$ for any $A \in \mathfrak{R}$. Thus we have

$$
s_{\psi}^{\tilde{P}}\left(\mathfrak{M}_{n}\right) \stackrel{s}{\longrightarrow} s_{\psi}^{\tilde{P}}\left(\mathfrak{M}_{\infty}\right)
$$

for any partition $\tilde{P} \in \mathscr{Q}(\mathfrak{M})$. Now we put

$$
h(\tilde{P}, \alpha) \equiv \sum_{j} \eta\left(\xi\left(P_{j}\right)\right)
$$

for any partition $\tilde{P} \in \mathscr{Q}(\mathfrak{M})$, then $h(\tilde{P}, \alpha)$ is bounded operator. Since $\xi(\cdot)=$ $E_{\varphi}\left(\cdot \mid \mathfrak{M}_{\infty}\right) \varphi$-a.e. for any $\varphi \in \mathfrak{S}_{I}(\alpha)$, we obtain

$$
h(\tilde{P}, \alpha)=s_{\varphi}^{\tilde{P}}\left(\mathfrak{M}_{\infty}\right) \quad \varphi \text {-a. e. }
$$

for any $\tilde{P} \in \mathscr{P}(\mathfrak{M})$ and $\varphi \in \widetilde{S}_{I}(\boldsymbol{\alpha})$. Finally the (2) of lemma 3 deduces the equality

$$
\varphi(h(\tilde{P}, \alpha))=S_{\varphi}^{\tilde{P}}\left(\mathfrak{M}_{\infty}\right)
$$

for any $\tilde{P} \in \mathscr{Q}(\mathfrak{M})$ and $\varphi \in \mathfrak{S}_{I}(\alpha)$.

Q.E.D.

Acknowledgments. The author wishes to express his gratitude to Prof. M. Ohya and Prof. H. Umegaki for their valuable suggestions and advices.

\section{REFERENCES}

[1] F. Hiai, M. Ohya And M. Tsukada, Sufficiency, KMS condition and relative entropy in von Neumann algebras. Paci. J. Math. 96 (1981), 99-109.

[2] F. Hial, M. Ohy a and M. Tsukada, Sufficiency and relative entropy in *-algebras with applications in quantum systems. Paci. J. Math. 107 (1983), 
117-140.

[3] M. Nakamura, and H. UMEgaki, A note on the entropy for operator algebras. Proc. Jap. Acad. 37 (1961), 149-154.

[4] M. Oнy A, Quantum ergodic channels in operator algebras. J. Math. Anal. Appl. 84 (1981), 318-327.

[5] M. Oнy A, Note on quantum probability. L. Nuovo Cimento, 38 (1983), 402-404.

[6] M. OнyA, Entropy transmission in $C^{*}$-dynamical systems. J. Math. Anal. Appl. 100 (1984), 222-235.

[7] M. Онуа, State change and entropies in quantum dynamical systems. Springer Lecture Notes in Math., 1136 (1985), 397-408.

[8] G.K. Pedersen, $C^{*}$-algebras and their Automorphism Groups. Academic Press, (1979).

[9] M. TAKESAKI, Tomita's Theory of Modular Hilbert Algebras and its Application. Lecture Notes in Math. Springer 128 (1970).

[10] H. UMEGAKI, Conditional expectation in an operator algebra. Tohoku Math. J. 6 (1954), 177-181.

[11] H. Umegaki, Conditional expectation in an operator algebra, II. Tohoku Math. J. 8 (1956), 86-100.

[12] H. UMEgAKI, Conditional expectation in an operator algebra, III. Kodai Math. Sem. Rep. 11 (1959), 51-64.

[13] H. UMEGAKI, Conditional expectation in an operator algebra, IV. (entropy and information). Kodai Math. Sem. Rep. 14 (1962), 59-85.

[14] H. UMEGAKI, General treatment of alphabet-message space and integral representation of entropy. Kodai Math. Sem. Rep. 16 (1964), 18-26.

[15] H. Umegaki And M. OHya, Entropy in Probabilistic Systems. Kyoritsu Shuppan, (1983), (in Japanease).

[16] H. UMEgaki AND M. OHya, Quantum Mechanical Entropy. Kyoritsu Shuppan, (1984), (in Japanease).

[17] H. Umegaki, M. Ohy a and F. Hiai, Introduction to Operator Algebras. Kyotitsu Shuppan (1985), (in Japanease).

DePARTMENT OF INFORMATION SCIENCES,

ScIence University of TOKyo. 